Original Research Paper

\title{
A Proposed Integrated Human Recognition for Security Reassurance
}

\author{
Zahraddeen Sufyanu, Fatma Susilawati Mohamad and Ahmad Salihu Ben-Musa
}

Faculty of Informatics and Computing, Universiti Sultan Zainal Abidin (UniSZA), 21300 Gong Badak Campus Terengganu, Malaysia

\author{
Article history \\ Received: 23-09-2014 \\ Revised: 01-10-2014 \\ Accepted: 30-03-2015 \\ Corresponding Author: \\ Zahraddeen Sufyanu \\ Faculty of Informatics and \\ Computing, Universiti Sultan \\ Zainal Abidin (UniSZA), \\ Terengganu, Malaysia \\ Email: sufyanzzz@yahoo.com
}

\begin{abstract}
A lot of systems require reliable and thorough authentication to ratify individual's claimed identity, especially in national and international security and defense. Unibiometric system suffers inherent weaknesses that are unavoidable in the system. Such inadequacies may directly or indirectly lead to an unacceptable error. For this reason, researchers pay great attention to the more advanced biometrics (referred to as multimodal biometrics). A multimodal biometric system involves at least two unimodal traits in a sole identification. This alleviates some of the drawbacks and improves recognition accuracy despite number of population considered. This research proclaims a new technique of integration for human recognition improvement using four physiological characteristics: Face, iris, palmprint and thumbprint. In addition, two fusion strategies namely, score-level and decision-level are presented using robust algorithms. The multimodal biometric systems possessed relative merits or pluses over its counterpart monomodal biometrics. The higher biometric modalities are integrated the more system is secured, hence better security assurance. Therefore, successfully conducting our research can derive supreme benefit over conventional multimodal biometrics. The integration results to outstanding gap coverage for security reassurance.
\end{abstract}

Keywords: Unibiometric, Multimodal Biometric, Integrated Human Recognition, Security Reassurance

\section{Introduction}

Biometrics is essentially a pattern recognition system (Ghandehari and Safabakhsh, 2011), which uses physiological and behavioral characteristics of a person; physiological characteristics may be fingerprint, iris, face or hand geometry (Bhattacharyya et al., 2009) and behavioral characteristics may be voice, signature, or other keystrokes dynamics. Human recognition is primarily used for verification or identification of an individual, for the purpose of security or authenticity. The recognition is either through the physiological or behavioral characteristics to ratify person's claimed identity. The application of statistical or structural techniques in analyzing those biological circumstances is referred to as biometric system or simply biometrics.

Single biometrics suffers limitations (Ross et al., 2006) such as lack of uniqueness, non universality of the chosen biometric trait and noisy data. Moreover, a single biometric often fails to be accurate enough for identification of large users and sometimes physical characteristics of a person for a selected bio metric might not be available or readable (Ko, 2005). Consequently, multimodal biometrics was introduced. The multimodal biometrics is simply combining two or more biometric features in a sole identification to achieve greater accuracy (Ko, 2005; Aggarwal and Gulati, 2012). Therefore, even if one modality is partially disturbed the other leads to an accurate identification. Multimodal system can eliminate these problems (Yang et al., 2007) and drastically minimize spoofs attacks; hence, improves the performance upon single biometrics (Jiaqiang et al., 2013). Biometric fusion can be combined at different levels viz. at data-sensor level, feature extraction level, matching-score level and decision-level (Ross and Jain, 2013; BWG, 2009). The justifiable reason of combining the two or more modalities is to improve the recognition accuracy (Aggarwal and Gulati, 2012) and overcome other limitations of single biometric systems (Nandakumar et al., 2008). 


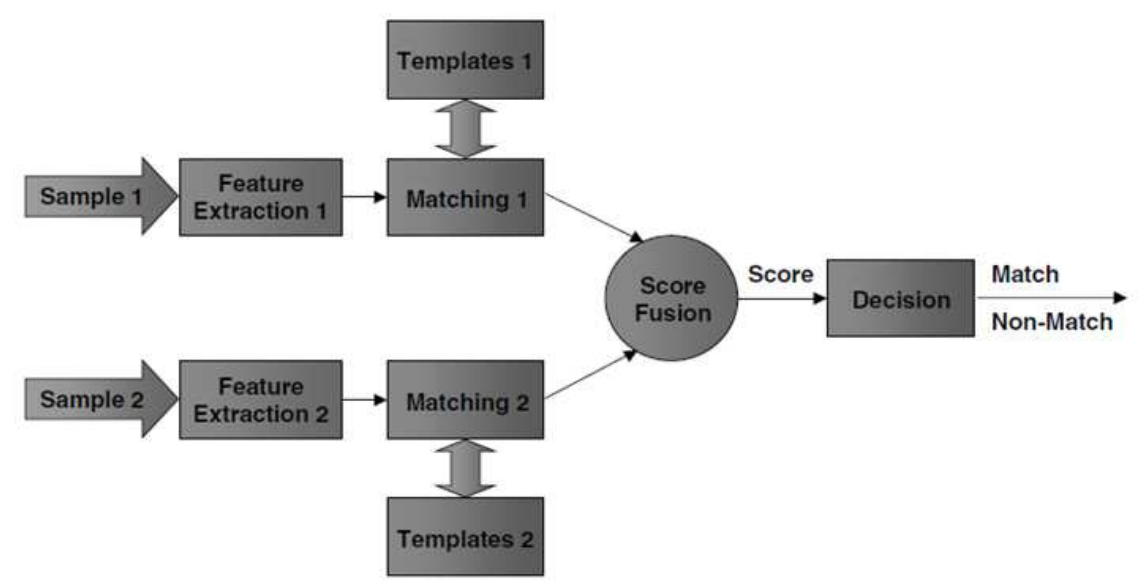

Fig. 1. Block diagram of multimodal system with score-level fusion (Raytheon, 2005)

In general (Imran et al., 2013) the fusion process enhances the system precision significantly which simply endorses well established fact about multimodal system.

In this study, the writers started with reviews of existing literature within previous decade, starting with single biometrics, further to a bimodal or pair wise biometrics and down to a tri-modal or compound biometrics. Significant improvement of multimodal biometrics over traditional, various methods of fusion, different fusion strategies and normalization practices and databases used where possible were reported in the review. Moreover, the paper covered mainly those reviews pertaining physiological characteristics of biometrics. Referring to the existing literature, there exist several integrated biometric schemes by various authors in the field of pattern recognition, employing different algorithms comprising; appearance based and texture based algorithms, neural network among others. Additionally, an integrated approach based human recognition for security reassurance is proposed. The multimodal biometric systems use various levels of fusion to combine two or more modalities (He et al., 2010).

Sensor Level: Fusion at the sensor level combines the two images from different sensors. Feature Extraction Level: Fusion at the feature level concatenates the features extracted using two or more modalities (Imran et al., 2013). Matching Score Level: Fusion at the matching score level combines the scores which describes the similarities of biometric traits acquired and their templates obtained by each biometric system. Or simply where the matching scores obtained from multiple matchers are combined. Decision Level: Fusion at the decision level combines the decisions taken by each biometric system, to obtain a final decision (Deriche, 2008) or simply where the accept/reject decisions of multiple systems are consolidated (Imran et al., 2013).
The flow chart depicting the multimodal system with score-level fusion is shown in Fig. 1.

The main reason of fusion these biometric traits is that, a system of highly demanding restriction such as border crossing control, national and international security and defense, need to systematically secure and guarantee user's absolute identity. Therefore, the main aim of this study is to cover an outstanding gap in multimodal biometric identification that will be used in the aforementioned areas. Thus implementing this system, can work efficiently for ensuring the security of systems.

The rest of the paper is organized as follows: Section 2, illustrated the review works of current biometric applications. The reviewed study is discussed in section 3. The proposed integrated approach is introduced in section 4. Conclusions and future scope are drawn in section 5.

\section{Review Works of Current Biometric Applications}

A number of biometric systems exist in various applications; each constitutes its strengths and weaknesses. Multimodal biometrics, as a successful application of biometric technology has recently receives significant attention by researchers to reveal new algorithms and efficient fusion techniques. In this section, several articles are investigated on conventional and modern approaches to biometrics; some of these literary works are categorized below:

\section{Single Modal Biometrics}

Single or sole biometrics involves the use of only one feature or biometric trait to form the recognition. It is the commonest biometric system exists in the literature and receives researchers' interest most, until now when trend of higher order biometrics supersedes. Few reviews under this category are described. 
Kaur et al. (2010) discussed a novel method of enhancing iris recognition by reducing complexity and increasing algorithm speed. The overall computational time was reduced from 7.016 to $1.109 \mathrm{sec}$ and reliability was also increased. Canny edge detection was used to generate an edge map and circle detection algorithm was used for iris localization. The iris was normalized, enhanced using local histogram equalization and filtered using Gaussian filter. For the feature extraction, cumulative sum based analysis method employed; finally Hamming Distance (HD) compared the similarity between the two iris codes during feature matching. The method reported accuracy of $99.38 \%$ and low FAR.

Asadi et al. (2010) compared the performance of face recognition using Principal Component Analysis (PCA) and Cross-Correlation Technique. Euclidean distance was used to find out the distance between two images. However, a database of different image sets of faces was constructed using two dimensions so that the covariance matrix will be $2 \times 2$. The face images of 12 people with different poses (48 images) were taken for testing. The recognition accuracies of PCA and CCA were $90 \% 85 \%$ respectively.

Zhonghua and Bibo (2010) proposed a method which recognized iris using Coefficients of Morlet Wavelet Transform, to enhance the efficiency of iris recognition and reduce complexity through iris localization, normalization for effective iris region. Different iris patterns were classified using pattern matching. A sum of 567 images from 81 subjects was used from CASIA iris database. The correct recognition rate reported using this method was $99.946 \%$.

Ghandehari and Safabakhsh (2011) evaluated the recognition performance of palmprint using statistical PCA and Adaptive Principal Component Extraction (APEX) method. The two algorithms were used to extract the principal component of the palmprint of 3000 palm-gray scale images on 300 users obtained from Hong Kong Polytechnic University (poly U.) palmprint database. Ten images were collected in two sections five for each section within interval of two months. The outputs were classified using Euclidean Distance (ED) and Hamming Distance (HD). The latter yielded better results than the former and APEX was reported to be faster.

Yanxia and Qiuqi (2006) demonstrated palmprint recognition system using Kernel Fisher Discriminant Analysis (KFDA) to extract higher order palmprint features for recognition. The work developed a scannerbased device that requires no fix pegs. KFDA was used to convert the nonlinear relations of input data and FLDA (fisherpalm) detected the linear relation and discriminate input features. The experiment considered ten images of both hands per individual and conducted on 80 people. KFDA was shown to be more effective than PCA (eigenpalms) and FLDA particularly with only few training samples.

Jacey-Lynn and Duncan (2011) investigated threedimensional face (3D) and facial expressions using Tensor based Multivariate Statistical Discriminant (TMSD) model, which employs multi linear algebra. The SUNY Binghamton 3D database from 100 subjects each with seven different facial expressions; neutral, anger, disgust, happiness; sadness, fear and surprise were considered. The face surfaces were preprocessed to produce a more robust recognition result. Euclidean distance scheme was finally used. The results of TMSD performed far better than PCA approach.

Samadi and Pourghassem (2013) introduced a practical algorithm for children faces classification from adults' using standard FG-NET database containing 1002 facial images of 82 different subjects, 411 and 591 were children and adults respectively. The faces were detected, landmarked and preprocessed. Statistical model of the face was carried out using face model parameters, LDA extracted the useful facial features from the model which resulted into a feature space, practically included the difference between adult and child. Lastly, Euclidean distance discriminant function categorized a child or an adult. Accuracy of $85 \%$ reported.

Wang et al. (2013) assessed images quality of palmvein and palmvein recognition with distance variations from the camera, also investigated the structural differences and clarity of Region of Interest (ROI) image and matched the result when palm changes from 3-8 cm. The palmvein ROI was evaluated using energy gradient function tenengrad. The results indicated that while palmvein becomes larger, the difference of image clarity and structure increases. FRR also increases.

Ahmad et al. (2011) compared face recognition using statistical and neural network techniques considering two parameters: Accuracy and recognition time. The paper explored Feed Forward Back Propagation-Neural Network (FFBP-NN), Generalized Regression Neural Network (GRNN), discriminant analysis and PCA. The experiments were conducted on ORL database containing 400 images from 40 persons, ten images per one, 13 females and 27 males. The neural network was proved better performance than the statistical.

Sangram and Davinder (2011) studied fingerprint recognition based on minutiae extraction process, involved preprocessing (i.e., image enhancement and image segmentation) and minutiae extraction. This was followed by minutiae-based matching technique, on the number of minutiae pairings between two finger prints to decide if finger matched or not. The input fingerprints were taken from FVC29004 database, the verification rate of the algorithm displayed about $65-70 \%$. FRR and FAR values were $30-38 \%$ approximately. 


\section{Pair Wise Biometrics}

Pair wise or bimodal biometric requires two biometric modalities to establish authentication or verification and to enhance improvement over single biometrics. Bimodal biometrics is very common in the literature; the performance can be improved by using efficient hybrid algorithms. Few reviews under this category are described.

Park et al. (2011) suggested a multimodal biometrics recognition system using fingerprint and vein. The approach used Local Binary Patterns (LBP) for fingerprint based on minutiae points and finger vein. Decision-level fusion of the fingerprint and finger vein was adopted. The result was reported having FAR of $0.019999 \%$ and FRR of $1.07 \%$. Significant improvement been achieved over single biometric system.

Ashraf et al. (2010) introduced a multimodal biometrics for face and fingerprint using Uniform Local Binary Patterns (ULBP) and minutiae extraction respectively. The format used matching score-level fusion for final recognition and obtained $99.4 \%$ accuracy. Individual result depicted $80 \%$ accuracy for the fingerprint and $97.6 \%$ for the face. The results were quite satisfactory.

Conti et al. (2010) created multimodal biometrics integration of iris and fingerprint using frequencybased approach and hamming distance based matching algorithm to achieve a unified homogeneous biometric vector. The paper adopted template-level fusion method and Log Gabor algorithm based codifier was used to obtain a unified template of both the fingerprint and the iris. For the similarity index computation HD was employed. Several results were achieved: Using FVC 2002 DB2B fingerprint database and randomly extracted same-size subset of BATH iris database. $\mathrm{FAR}=0 \%$ and $\mathrm{FRR}=5.71 \%$ were observed. In addition, using BATH and FVC 2002 DB2A another interesting working point of $\mathrm{FAR}=0 \%$ and $\mathrm{FRR}=7.28 \% / 9.7 \%$ was reported.

Wang et al. (2013) suggested a feature level fusion of face and palmprint using Canonical Correlation Analysis (CCA) and PCA for dimension reduction. The combined canonical correlation characteristics were extracted before the features were normalized to eliminate unbalance. Euclidean distance was used for recognition. The experiment was conducted on ORL face database and poly $U$ multispectral palmprint database. The paper also compared the performance of PCA, other fusion techniques: Serial rule, sum rule, weighted sum rule and complex field with the proposed method. The new method showed lowest EER.

Tekade (2012) discussed a multimodal recognition of face and ear using feature fusion of average rule and PCA scheme. 400 face images from ORL database and 375 images from IIT Delhi ear database were utilized.
Fisher linear discriminant analysis was used for feature extraction of the face and the ear separately whereas Euclidean distance for comparison. The paper compared the results of average rule and that of PCA fusion algorithm. PCA acquired better performance.

$\mathrm{Xu}$ et al. (2013) Initiated a novel joint face and iris multimodal biometric recognition using Contourlet Transform (CT) and two directional two Dimensional $(2 \mathrm{D})^{2}$ PCA. The former was used to extract the iris feature and the latter to extract the face feature. The experiment was conducted using AR face database and CASIA iris database. The proposed joint method outweighed the performance of the single face and iris modal, under all varying conditions of training and Testing Sample Ratio (TTR).

Ahmad et al. (2010) evaluated a multimodal system for face and palmprint recognition. Gabor based image processing was utilized to extract discriminant features, while PCA and LDA were used to reduce the dimension of each modality. The multimodal fusion at feature level of face and palmprint produced better recognition result (99.5\%) compared to single modal biometrics method (94\% for fisherpalm $87 \%$ for eigenfaces, $92 \%$ for fisherfaces and $91.5 \%$ for eigenpalm).

This recent paper by Imran et al. (2013) was an addition to their earlier paper in (2010). They reintroduced a multimodal biometrics based on fusion of face and palmprint modalities at all levels of fusion and different fusion strategies using popular appearance based algorithms and texture based feature extraction algorithms. LDA and LPQ algorithms were selected to extract the features of face and palmprint respectively. The experiments were conducted on AR face database and poly U. palmprint database under clean and noise conditions and several observations were made as we will later see in our discussion.

Deshmukh et al. (2013) proposed a biometric authentication system based on feature level fusion of face and fingerprint using Gabor filter bank to extract the features independently. PCA and LDA were used for dimension reduction and discriminating enhancement. Distance classifiers authenticate the results. The performance was expressed using ORL face database and FVC 2002 fingerprint database. The proposed system reduced FAR and FRR to 0.35 and $0.75 \%$ respectively and achieved recognition accuracy of $99.25 \%$.

Faten et al. (2013) set up a bimodal biometrics which integrates face and fingerprint for user identification using Gabor wavelet. It was conducted on ORL face database and FVC 2004 fingerprint database. PCA extracted the eigenvectors for the face recognition and minutiae technique for the fingerprint. The multimodal reduced equal error rate and enhanced performance over single biometrics. 


\section{Compound Biometrics}

Compound biometrics involves more than two evidences presented by different traits belongs to a user to form their identity as a whole and enhance authenticity efficiency. Although the system might be complex and cost higher due to the requirement of new sensors and longer time during recognition. Consequently, the performance can be significantly improved by utilizing more than one trait for a sole recognition. To the best of the authors' knowledge triple biometrics (where three biometric modalities are considered) is the highest biometrics exists in the literature. Few reviews under this category are described.

Hanmandlu et al. (2010) investigated a multimodal biometric system based on hand traits namely, hand geometry, palmprint and handveins at score-level fusion using t-norm strategy. The experimental results discovered that the score-level approach using t-norm rendered fairly good performance and did not require any iteration. The proposed fusion using Hamacher tnorm yielded Genuine Acceptance Rate (GAR) of 99.9\% at FAR of $0 \%$. Therefore, good improvement over individual biometrics was observed.

Vivek et al. (2012) Described feature extraction techniques of fusion fingerprint, iris and face using Gaussian Mixture Model (GMM). Expectation Maximization method was used to obtain the parameters needed to implement the GMM. The fingerprint was extracted using minutiae technique. For the iris, segmentation, normalization and feature encoding were accomplished. For the face PCA was used. The fingerprint, iris and face images were collected from FBI, poly and Sino biometrics database respectively and information were fused at match score-level using a density based score level fusion. GMM gave superior results.

Yaghoubi and Eliasi (2011) proposed a multimodal biometrics using face, ear and palm based on feature extracted from visual cortex, achieved through transforming face and palmprint images with Gabor filter and ear images with Gaussian filter in HMAX method. However, matching score level fusion was employed. The classification was done using K-NN and SVM classifiers and the experimental results showed 96\% accuracy rate on ORL face database, 94\% accuracy rate on USTB Ear database and $96.6 \%$ accuracy rate on POLYU Palm database. Recognition time was not mentioned which is another major concern in multimodal system.

Dinakardas et al. (2013) presented a multimodal identification system based on face, fingerprint and iris. Performance of two different models on these biometric traits was evaluated. The experiments were conducted on a real database consisting 500 persons. The first model extracted fingerprint and iris features using PCA while face using fisherfaces. The second model extracted fingerprint using minutiae approach, iris using LBP and maintaining fishefaces for the face. The results were depicted under ROC curve, standard deviation and 95\% confidence interval. The single classifier approach of minutiae based fingerprint extraction and LBP based iris extraction depicted better result than when PCA was used. The multimodal classifier configuration of the second approach outperformed the first.

Imran et al. (2011) introduced a new hybrid approach multi biometrics of fusion face and iris using three algorithms: Independent Component Analysis1 (ICA1), PCA and Local Phase Quantization (LPQ) based on different level of fusion. It also discussed comparative analysis of the proposed method with multi-algorithmic and multimodal approaches. The results from the experiments were based on considering all level of fusion excluding sensor level and conducted on ORL-face, CASIA-iris and FVC-2006 fingerprint database. The performance was compared using EER and the proposed hybrid method outperformed the other approaches.

This paper of Xiuyan et al. (2011) is based on multimodal biometric of hand vein, iris and fingerprint. Theoretical and experimental studied were compared. Mathematical model of matching score was deduced and score normalization algorithms were analyzed and particularized. Simple Average (SA) and Weighting Average (WA) fusion algorithms were analyzed too. The biometric recognition experiments were assessed on TJU hand vein database, CASIA iris and fingerprint database to verify the fusion theory. The results indicated that the experiments data were consistent with the deduced theoretic results.

Yazdanpanah et al. (2010) instigated a multimodal biometric identification system based on features extracted from three biometric modalities including face, ear and gait using Gabor and PCA. Fusion at matching score was performed on ORL face database, USTB ear database and CASIA gait database. The paper evaluated three different kinds of normalization techniques experimentally and two kinds of fusion methods. Z-score method of normalization combined with weighed product method of fusion gave the best recognition performance of $97.5 \%$ at $0.1 \%$ FAR. The innovative approach outperformed the unimodal systems on a variety of image databases.

Ko (2005) investigated different levels of fusion and fusion strategies that can be adopted in multimodal biometrics. Meanwhile the possible scenarios in multimodal biometric system using finger, face and iris recognition and how they affect the overall identification accuracy were discussed. The need of staffing levels to properly operate the system was also mentioned.

Chaudhary and Nath (2009) suggested a multimodal biometric recognition based on fusion of palmprint, 
fingerprint and face at matching score-level. The fusion module performed score normalization using min-max rule before the three normalized scores with their individual weights were combined into a total score by sum rule. The total scores was passed to the decision module and finally declared a person as genuine or an impostor. Both the existing biometric system and the multimodal system were plotted on Genuine Accept Rate (GAR) against False Accept Rate (FAR). The proposed system gave better performance.

Yang and Ma (2007) developed a new fusion technique of identifying fingerprint, palmprint and handgeometry. The work proposed the use of feature fusion and matching score-level fusion for the identification. The former fused fingerprint and palm-print to form a Joint Feature Vector (JFV) and the latter fused the JFV and the hand-geometry. The qualities of the images were measured using Wavelet Scalar Quantization (WSQ) method. The experiments were realized using Mat lab language and tested on a database of 98 persons. The result proved the feasibility of the approach and this method was highly investigated.

\section{Discussion and Comparison of Techniques}

In the enhanced iris recognition method by (Kaur et al., 2010) using cumulative sum based analysis method, substantial accuracy improvement was witnessed over the existing method and achieved FAR of 0.01 compared to 0.05 in the previous one and excessively reduced overall operating time. Fingerprint extracted based on minutiae process obtained verification rate of $65-70 \%$. The low recognition rate was due to inefficient algorithm used and poor quality of images in the database (Sangram and Davinder, 2011). APEX which is one of the PCA techniques involving neural network, though affected by initialization of the network but was more efficient than statistical PCA especially with HD. However, the recognition performance was increased with increased in training samples in both the algorithms (Ghandehari and Safabakhsh, 2011). The statistical model algorithm mentioned in (Wang et al., 2007) for children faces classification showed good result despite classifying images that were opaque. Meanwhile, when neural network classifier was applied (Samadi and Pourghassem, 2013) with different amount of specifications to discriminate child faces, the result was not bettered. The proposed approach for fusion of face, ear and palm illustrated in (Yaghoubi and Eliasi, 2011) showed great performance using score-level fusion and SVM outclassed KNN. The recognition rate of KFDA and FLDA were much higher than PCA whereas FLDA (fisherpalms) performed lesser than KFDA because it cannot describe complicated nonlinear variations such as rotation, movement and stretching variations of hands, but it was a good feature selector since increasing the number of training samples, fisher palms recognition rate approaches KFDA closely (Yanxia and Qiuqi, 2006). Consequently, FLDA needs to be verified using dissimilar large samples. The performance of TMSD and PCA were compared using Euclidean distance scheme for recognition. The advantage of this classifier is its high speed (Samadi and Pourghassem, 2013). PCA (represents the principal axes of variations across the face) while TMSD (locates the principal axes of variation across various facial expressions and reports the various expression factors related to one another). The recognition of the former surpassed the latter. The low recognition rates for faces with surprise expressions were due to the extreme geometric changes. The proposed method was not effective at distinguishing facial expression that appears similar, such as anger and disgust, fear and surprise as excerpted from (Jacey-Lynn and Duncan, 2011).

Identification of face, fingerprint and iris in Dinakardas et al. (2013), LBP based iris extraction couple with minutiae for fingerprint displayed better result than the use of PCA in their feature extractions. Conversely, PCA was used for dimension reduction with canonical correlation analysis in Wang et al. (2013) and better performance reported. Also when PCA scheme and average rule were used for feature level fusion with LDA as feature selector in Tekade (2012), the PCA outweighed the average rule and better performance reported. The paper by Wang et al. (2013) only emphasized extraction of good quality image in contactless recognition so that each user can simply raise their palms closer to the scanner, no experimental results for comparison of the distance variations results of palmvein recognition. On the other hand, Yanxia and Qiuqi (2006) avoided inconveniencing user by developing a scanner-based device which makes no use of fix pegs; this idea is more cogent and can be practically implemented. It was evidently shown in Ahmad et al. (2011) on face recognition that GRNN performed best, followed by Discriminant Analysis (DA), PCA, BP-NN in this order and in terms of consumed time during recognition, DA was proved to be the best technique. Among the popular appearance based algorithms (PCA, LPP, ICA1, LDA) LDA was discovered best for face and among the popular texture based feature extraction algorithms (LPQ, LBPV, LogGabor) significant improvement was observed by LPQ for palmprint in (Imran et al., 2013). Similar efforts by the same authors in (2011) LPQ, PCA and ICA1 were selected for their new hybrid method and the performance measured in terms of EER. Additionally when wavelet decomposition scheme applied at sensor level fusion, the performance was not satisfactory, it was even worse than the unimodal counterparts. The bad result was presumed since fusion at the sensor level or feature level is not always possible due to the 
heterogeneity or incompatibility of the information content (Jain and Arun, 2007) and fusion at feature extraction level is difficult because feature vectors which are used by different biometric traits may be inaccessible or incompatible (Yazdanpanah et al., 2010). Although after these claims, there were many research on feature fusions which were successful, some reported in this study. In any way, due to easily accessing and easily integrating, fusion at matching score level is generally preferred.

Normalization includes mapping the scores obtained from each modality into a common domain. A good normalization method should be robust and efficient. Zscore outperformed Min-max and Median-MAD normalization techniques in that paper and obtained excellent recognition, so also the weighted Product which involves higher computational cost performed better than the weighted sum. At the same time, the Zscore normalization and tanh normalization scheme were reported having similar performance under clean data, though tanh normalization scheme obtained robust result when corrupted with noise. Z-score model is the most commonly used at present, this kind of model uses the arithmetic mean and variance of the given data to normalize the element in the set. The mean value is 0 and the standard deviation is 1 after the normalization. The fusion performed at score-level, adopting the sum rule, obtained great results under both clean and noise conditions and it was mentioned that the results may vary based on type of feature extraction algorithms employed. Feature level fusion strategies of min-max and Z-score showed minimum EER with Z-score.

Face and palmprint is preferable in many multimodal biometric applications. The score level fusion of face and palmprint adopting the sum rule obtained best results compared to min score and max score rules. In the 'Sum rule' the contribution made by individual matcher will be considered for decision making. In slightly similar way, a weighed sum rule which is one of the parallel fusion methods performed better than ordinary sum rule in Wang et al. (2013). Progressively, coming to decision level fusion, 'OR rule' outperformed 'AND rules' in many literatures. It is primarily due to the reason that if any one of the matchers classifies a test sample genuine, the decision will be regarded as genuine. The theoretical significance of multimodal biometric recognition based on classic fusion algorithm at feature matching level was described in Xiuyan et al. (2011), since most of the existing literatures were reintroducing fusion algorithm and verify their novel method by experiments again and again.

\section{Significance of the Study}

The major aim of reviewing the literature is to arrest the failure of single biometrics, the insufficiency of pair wise biometrics and improve triple biometrics, since many studies have recommended multimodal biometrics for current and future applications. Multimodal biometric systems own ability to withstand spoof attacks, while single biometric sometimes fails to extract adequate information for verifying the identity of a person. Due to the presence of multiple, fairly independent pieces of evidence, multi-biometrics are expected to be reliable (Jain et al., 2004) and enhanced performance reliability could be achieved by combining multiple modalities. The biometrics test studied in NIST (2002) has revealed that, to achieve acceptable identification accuracy for large user applications multimodal biometrics is required. The justifiable reason of combining the two or more modalities is to improve the recognition accuracy $(\mathrm{Ko}, 2005)$ and that is very possible when features of independent biometrics are statistically combined. Combining different modalities produces a system which can outperform single modalities (Kittler and Hojjatoleslami, 1998).

Several methods have been proposed by researchers, on the multimodal biometrics, such methods were reported to have significance improvement over traditional biometrics traits. The performance of a system can be improved by utilizing more than one trait (Ravi and Dattatreya, 2013), though the cost of the system is substantially higher due to requirement of new sensors (Hanuma, 2011). The application of higher integrated biometric system in a highly security demanding applications will be remarkable. The higher the number of features integrated, the more system is secured, hence better system protection. Therefore, successfully conducting our research can derive supreme benefit over conventional multimodal biometrics.

Another reason for carrying out the review in the previous section is to allow the writers choose the best algorithm for their proposed approach, since the aim of any biometric system is to improve recognition accuracy. This is normally achieved by comparing different existing algorithms on a specific problem and selects the best among (Imran et al., 2010). But selecting the best algorithm is not an easy task, because an algorithm may work under certain condition, but not suitable or compatible on another condition or problem. In addition, no algorithm ever exists without any limitation. LDA is the most dominant algorithm for feature selection in appearance based method, it yields an effective representation that linearly transforms the original data space into a low-dimensional feature space where the data is well separated (Mohammed and Gupta, 2013). The dataset selected should have larger samples per class for good extraction of discriminating features. Under the test and comparison of performance of appearance based statistical methods on colored face images in (Önsen, 2003) LDA surpassed ICA under different illuminations and more sensitive than PCA 
and ICA on partial occlusions. LDA has small size problem when dealing with high dimensional data (Swets and Weng, 1996). It defines a projection that makes the within-class scatter small and the betweenclass scatter large (Belhumeur et al., 1997) and trying to find non-linear correlation models among different biometric features can improve the system and recommended that, the intra-class correlation for different feature set should be further explored on extended biometric data sets (Soviany and Puscoci, 2013).

The four physiological biometrics employed in our research have receive significant benefit, since palmprint is user friendly, robust and accurate. Iris texture is unique even for twins. Fingerprint and face have been reliable methods as reported in lots of literature. The model provides an understanding ground between the users and system providers, since the users' identity are totally secured. High priority of the system is; the model will effectively be applied to a real world problem in order to ease security personnel and improve efficiency of identification, by disallowing any fraudulent act.

\section{Proposed Technique}

The study proposed a new integrated biometrics approach using four different modalities namely, face, palmprint, iris and thumbprint applying two fusion strategies of score-level and decision-level. Appearance based techniques will be used as feature selectors. The result of the feature extraction called feature vectors are fed into the separate matching modules. Each matching module compares the feature vectors against the database using Euclidean distance classifier, to measure the similarities between the test vectors and the reference vectors in the gallery.

The pair matched scores of the bimodal outputs of iris and thumb, face and palm are regularized using Z-score normalization scheme. The normalized scores are combined into total scores at fusion modules 1 and 2 using weighted sum rule. These are fed to the separate decision modules 1 and 2, where the scores are classified as correlated or uncorrelated using cross correlation analysis and rule is applied at final decision-fusion so that both matchers have to deem the test samples as genuine to classify a person as genuine otherwise as an imposter.

This is computed by comparing these final scores with a threshold. The novelty of this approach is the quadruple biometrics integration, using robust bi-fusion techniques and deducing a mathematical modeling of integration to check the consistency of the experimental figures. To the best of the writers' knowledge, there has never existed such approach in the literature.

The framework of the proposed multimodal biometric system based on matching score and decisionlevel fusion is shown in Fig. 2.

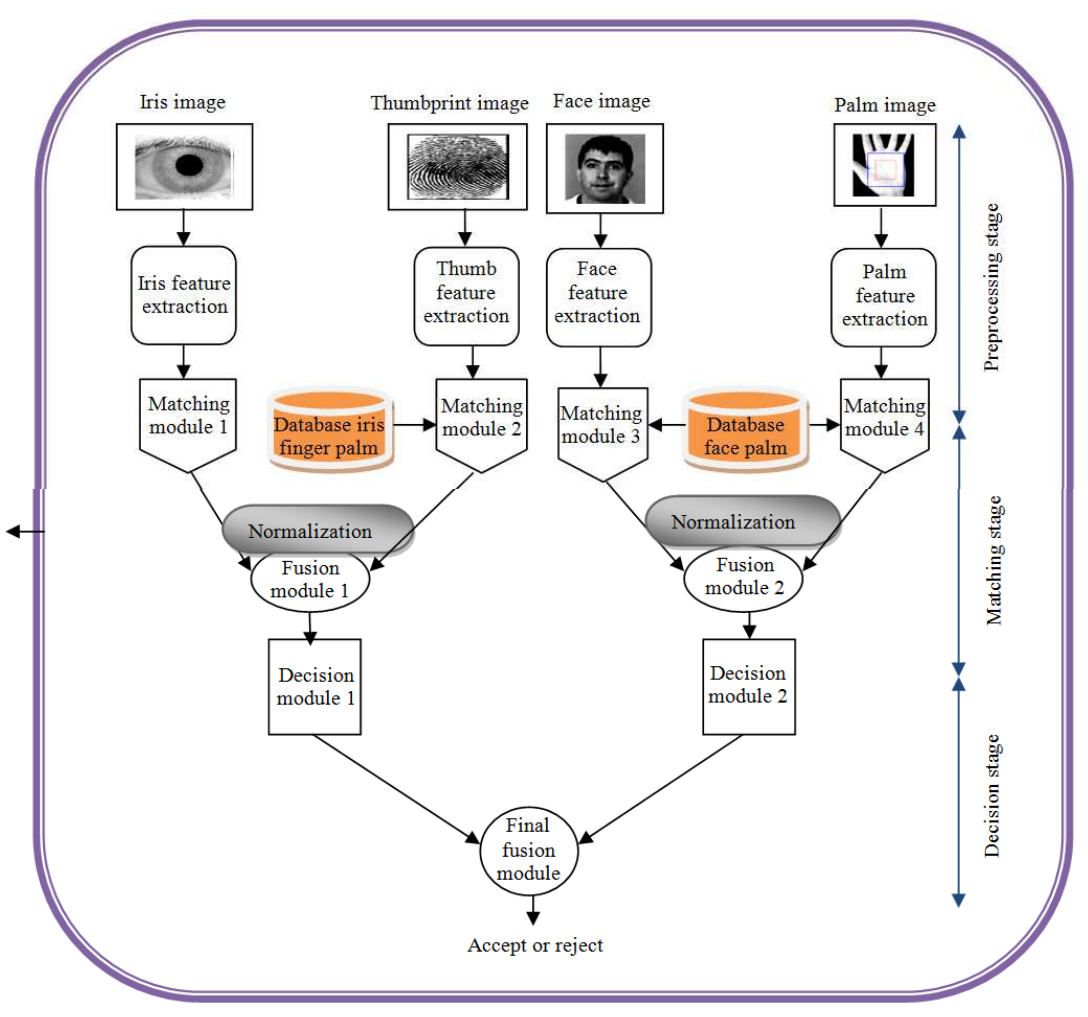

Fig. 2. A framework of the proposed score/ decision-level fusion 


\section{Conclusion}

The paper reviewed most recent developments on biometric technology and their comparative pluses over conventional biometrics. Very many literatures have concluded multimodal biometric system as the best method to prevent spoof attacks, since an impostor can hardly spoof genuine user multiple biometry features simultaneously. The essay proposed integrated approach of fusion face, iris, palm and thumbprint features to form a sole recognition system, so as to highly protect users' identity in extremely restricted environments. However, to investigate the experimental contention of the approach and its influence to security reassurance, a simple but robust means of fusion algorithms is considered, so that training the user how to use the system will not be complicated. Furthermore, more statistical algorithms and classifiers will be exploited to intensively prove the benefit of this research and pronounce the most robust algorithms in the literature. Finally, we hope to see in the subsequent chapters following this research, the theoretical model of the integration to buttress the proposed method.

\section{Acknowledgement}

This work was conducted and financially supported by University Sultan Zainal Abidin (UniSZA), Kuala Terengganu, Terengganu, Malaysia under the Grant No (UniSZA/14/GU/019). The corresponding author thanks the Kano State Government Nigeria, for providing the scholarship.

\section{Author's Contributions}

Fatma Susilawati Mohamad: This author suggested the proposed concept and contributed to the analysis of the design and was aware of all the preparation of this manuscript and gave final approval of the final version to be submitted.

Ahmad Salihu Ben-Musa: This author participated in reviewing some of the related works and assisted in drafting and revising the article critically to ensure the manuscript followed good structure. He also gave final approval of all the versions to be submitted.

Zahraddeen Sufyanu: This author made substantial contributions to conception and design and in handling the revised manuscript. However, he interpreted the new idea with intellectual discussions.

\section{Ethics}

The authors strive to ensure that the work provides novelty in technical innovation and it has never been published elsewhere. The design criteria will be well assessed in future studies to avoid any risk in implementation of this approach. All authors are accountable for any revised version. Therefore, no conflict of interest declared.

\section{References}

Aggarwal, S. and Y. Gulati, 2012. A multimodal biometric system using fingerprint and face. Int. J. Adv. Res. Comput. Eng. Technol., 1: 966-703.

Ahmad, M.I., W.L. Woo and S.S. Dlay, 2010. Multimodal biometric fusion at feature level: Face and palmprint. Proceedings of the 7th International Symposium on Communication Systems Networks and Digital Signal Processing, Jul. 21-23, IEEE Xplore Press, Newcastle upon Tyne, pp: 801-805.

Ahmad, T., A. Jameel and B. Ahmad, 2011. Pattern recognition using statistical and neural techniques. Proceedings of the International Conference on Computer Networks and Information Technology, Jul. 11-13, IEEE Xplore Press, Abbottabad, pp: 87-91. DOI: 10.1109/ICCNIT.2011.6020913

Asadi, S., D.V.S. Rao and V. Saikrishna, 2010. A comparative study of face recognition with principal component analysis and crosscorrelation technique. Int. J. Comput. Applic., 10: 17-21. DOI: 10.5120/1502-2019

Ashraf, A., M.Z. Walaa, M.S. Omar, M.N. Nadia and S. Gerald, 2010. Human authentication using faee and fingerprint biometries. Proceedings of the 2nd International Conference on Computational Intelligence, Communication Systems and Networks, (CSN' 10), pp: 274-278.

Belhumeur, P.N., J.P. Hespanha and D. Kriegman, 1997. Eigenfaces Vs. Fisherfaces: Recognition using class specific linear projection. IEEE Trans. Pattern Analysis Machine Itellig., 19: 711-720. DOI: $10.1109 / 34.598228$

Bhattacharyya, D., R. Ranjan, A.F. Alisherov and M. Choi, 2009. Biometrie authentieation: A review. Int. J. u-and e-Service, Sci. Technol., 2: 13-27.

BWG, 2009. Biometrics Security Concerns. UK Biometrics Working Group.

Chaudhary, S. and R. Nath, 2009. A multimodal biometric recognition system based on fusion of palmprint, fingerprint and face. Proceedings of the International Conference on Advances in Recent Technologies in Communication and Computing, Oct. 27-28, IEEE Xplore Press, Kottayam, Kerala, pp: 596-600. DOI: 10.1109/ARTCom.2009.224

Conti, V., C. Militello and F. Sorbello, 2010. A frequency-based approach for features fusion in fingerprint and iris multimodal biometric identification systems. IEEE Trans. Syst. Man, Cybernetics-Part C: Applic. Rev., 40: 384-395.

DOI: 10.1109/TSMCC.2010.2045374 
Deriche, M., 2008. Trends and challenges in mono and multi biometrics. Proceeding of the 1st Workshops on Image Processing Theory, Tools and Applications, Nov. 23-26, IEEE Xplore Press, Sousse, pp: 1-9. DOI: 10.1109/IPTA.2008.4743801

Deshmukh, A., S. Pawar and M. Joshi, 2013. Feature level fusion of face and fingerprint modalities using Gabor filter bank. Proceedings of the International Conference on Signal Processing, Computing and Control, Sept. 26-28, IEEE Xplore Press, Solan, pp: 1-5. DOI: 10.1109/ISPCC.2013.6663404

Dinakardas, C., S.P. Sankar and G. Nisha, 2013. A multimodal performance evaluation on two different models based on face, fingerprint and iris templates. Proceedings of the International Conference on Emerging Trends in VLSI, Embedded System, Nano Electronics and Telecommunication System, Jan. 79, IEEE Xplore Press, Tiruvannamalai, pp: 1-6. DOI: 10.1109/ICEVENT.2013.6496558

Faten, B., B. Mossaad and L. Kais, 2013. Multimodal biometric identification system based on face and fingerprint. Proc. Eng. Technol., 3: 219-222.

Ghandehari, A. and R. Safabakhsh, 2011. A comparison of principal component analysis and adaptive principal component extraction for palmprint recognition. Proceedings of the International Conference on Hand-Based Biometrics, Nov. 17-18, IEEE Xplore Press, Hong Kong, pp: 1-6.

DOI: 10.1109/ICHB.2011.6094307

Hanmandlu, M., J. Grover, V.K. Madasu and S. Vasirkala, 2010. Score level fusion of hand based biometrics using t-norms. Proceedings of the International Conference on Technologies for Homeland Security, Nov. 8-10, IEEE Xplore Press, Waltham, MA, pp: 70-76.

DOI: $10.1109 /$ THS.2010.5655093

Hanuma, M., 2011. Real-time live face detection using face template matching and DCT energy analysis. Proceedings of the International Conference of Soft Computing and Pattern Recognition (CPR' 11), pp: 342-346.

He, M., S.J. Horng, P. Fanc, R.S. Rund and R.J. Chend et al., 2010. Performance evaluation of score level fusion in multimodal biometric systems. Pattern Recognit., 43: 1789-1800.

Imran, M., A. Rao and G.H. Kumar, 2010. Multibiometric systems: A comparative study of multi-algorithmic and multimodal approaches. Procedia Comput. Sci., 2: 207-212.

DOI: 10.1016/j.procs.2010.11.026

Imran, M., A. Rao and G.H. Kumar, 2011. A new hybrid approach for information fusion in multibiometric systems. Proceedings of the 3rd National Conference on Computer Vision, Pattern Recognition, Image Processing and Graphics, Dec. 15-17, IEEE Xplore Press, Hubli, Karnataka, pp: 235-238. DOI: 10.1109/NCVPRIPG.2011.57
Imran, M., A. Rao and G.H. Kumar, 2013. Multimodal biometric fusion of face and palmprint at various levels. Proceedings of the International Conference on Advances in Computing, Communications and Informatics, Aug. 22-25, IEEE Xplore Press, Mysore, pp: 1793-1798.

DOI: 10.1109/ICACCI.2013.6637453

Jacey-Lynn, M. and D. Gillies, 2011. A tensor-based multivariate statistical model for 3D face and facial expression recognition. Proceedings of the 7 th International Conference on Information Technology in Asia, Jul. 12-13, IEEE Xplore Press, Kuching, Sarawak, pp: 1-8. DOI: $10.1109 /$ CITA.2011.5998383

Jain, A.K. and R. Arun, 2007. Handbook of Multimodal biometrics. Springer.

Jain, A.K., A. Ross and S. Prabhakar, 2004. An introduction to biometric recognition. IEEE Trans. Circuits Syst. Video Technol., 14: 4-20. DOI: $10.1109 /$ TCSVT.2003.818349

Jiaqiang, W., Y. Ming, Q. Hanbing and L. Bin, 2013. Analysis of palm vein image quality and recognition with different distance. Proceedings of the Fourth International Conference on Digital Manufacturing and Automation Jun. 29-30, IEEE Xplore Press, Qingdao, pp: 215-218.

DOI: 10.1109/ICDMA.2013.50

Kaur, G., A. Girdhar and M. Kaur, 2010. Enhanced iris recognition system-an integrated approach to person identification. Int. J. Comput. Applic., 8: 1-5. DOI: $10.5120 / 1182-1630$

Kittler, J. and S.A. Hojjatoleslami, 1998. A weighted combination of classifiers employing shared and distinct representations. Proceedings of the IEEE Computer Society Conference on Computer Vision and Pattern Recognition, Jun. 23-25, IEEE Xplore Press, Santa Barbara, CA, pp: 924-929. DOI: $10.1109 /$ CVPR.1998.698715

Ko, T., 2005. Multimodal biometric identification for large user population using fingerprint, face and iris recognition. Proceedings of the 34th Applied Imagery and Pattern Recognition Workshop, Dec. 1-1, IEEE Xplore Press, Washington, DC, pp: 223-228. DOI: 10.1109/AIPR.2005.35

Mohammed, J. and B. Gupta, 2013. Performance comparison of various face detection techniques. Int. J. Sci. Res. Eng. Technol., 2: 46-52.

Nandakumar, K., Y. Chen, S.C. Dass and A. Jain, 2008. Likelihood ratio-based biometric score fusion. IEEE Trans. Pattern Analysis Mach. Intellig., 30: 342-347. DOI: 10.1109/TPAMI.2007.70796.

NIST, 2002. Summary of NIST standards for biometric accuracy, tamper resista NCE and interoperability.

Önsen, T.A.A., 2003. Face recognition using PCA, LDA and ICA approaches on colored images. J. Electrical Electron. Eng. 
Park, Y.H., D.N. Tien, E.C. Lee, K.R. Park and H.C. Kim, 2011. A multimodal biometric recognition of touched fingerprint and finger-vein. Proceedings of the International Conference on Multimedia and Signal Processing, May 14-15, IEEE Xplore Press, Guilin, Guangxi, pp: 247-250.

DOI: $10.1109 /$ CMSP.2011.57

Ravi, S. and P. Dattatreya, 2013. Multimodal biometric approach using fingerprint, face and enhanced iris features recognition. Proceedings of the International Conference on Circuits, Power and Computing Technologies, Mar. 20-21, IEEE Xplore Press, Nagercoil, pp: 1143-1150. DOI: 10.1109/ICCPCT.2013.6528884

Ross, A. and A. Jain, 2003. Information fusion in biometrics. Pattern Recogn. Lett., 24: 2115-2125. DOI: 10.1016/S0167-8655(03)00079-5

Ross, A.A., K. Nandakumar and A.K. Jain, 2006. Handbook of Multibiometrics. 1st Edn., Springer Science and Business Media, New York, ISBN-10: 0387331239, pp: 220.

Samadi, A. and H. Pourghassem, 2013. Children detection algorithm based on statistical models and LDA in human face images. Proceedings of the International Conference on Communication Systems and Network Technologies, Apr. 6-8, IEEE Xplore Press, Gwalior, pp: 206-209.

DOI: 10.1109/CSNT.2013.52

Sangram, B. and K. Davinder, 2011. Fingerprint recognition using image segmentation. Int. J. Adv. Eng. Sci. Technol., 5: 012-023.

Soviany, S. and S. Puscoci, 2013. A feature correlationbased fusion method for fingerprint and palmprint identification systems. Proceedings of the 4th International Conference on E-Health and Bioengineering-EHB, Nov. 21-23, IEEE Xplore Press, Iasi, 1-4. DOI: 10.1109/EHB.2013.6707259

Swets, D.L. and J.J. Weng, 1996. Using discriminant eigenfeatures for image retrieval. IEEE Trans. Pattern Analysis Mach. Intellig., 18: 831-836. DOI: $10.1109 / 34.531802$

Tekade, A.A., 2012. Feature fusion method based on fisher discriminant analysis for face and ear for multimodal recognition. Int. J. Eng. Res. Technol.

Vivek, S.A., J. Aravinth and S. Valarmathy, 2012. Feature extraction for multimodal biometric and study of fusion using Gaussian mixture model. Proceedings of the International Conference on Pattern Recognition, Informatics and Medical Engineering, Mar. 21-23, IEEE Xplore Press, Salem, Tamilnadu, pp: 387-392. DOI: 10.1109/ICPRIME.2012.6208377

Wang, J.G., W.Y. Yau and A. Suwandy, 2007. Fusion of palmprint and palm vein images for person recognition based on "Laplacianpalm" feature. Proceedings of the Conference on Computer Vision and Pattern Recognition, Jun. 17-22, IEEE Xplore Press, Minneapolis, MN, pp: 1-8.

DOI: 10.1109/CVPR.2007.383386
Wang, Z., C. Liu, T. Shi and Q. Ding, 2013. Face-palm identification system on feature level fusion based on CCA. J. Inform. Hiding Multimedia Signal Process., 4: 272-279.

Xiuyan, L., M. Changyun L. Tiegen and Y. Chenhu, 2011. Theoretical analysis and experimental study on multimodal biometric. Proceedings of the International Conference on Control, Automation and Systems Engineering, Jul. 30-31, IEEE Xplore Press, Singapore, 1-4. DOI: 10.1109/ICCASE.2011.5997781

Xu, Y., F. Luo, Y. Xu and Y. Zhai, 2013. Multi-modal biometric recognition algorithm based on iris and facial images. J. Comput. Inform. Syst., 9: 6743-6750.

Yaghoubi, Z. and M. Eliasi, 2011. Robust biometric authentication based on feature extracted from visual ventral stream. Proceedings of the IEEE International Conference on Computer Applications and Industrial Electronics, Dec. 4-7, IEEE Xplore Press, Penang, pp: 448-452.

DOI: 10.1109/ICCAIE.2011.6162177

Yang, F. and B. Ma, 2007. A new mixed-mode biometrics information fusion based-on fingerprint, hand-geometry and palm-print. Proceedings of the 4th International Conference on Image and Graphics, Aug. 22-24, IEEE Xplore Press, Sichuan, pp: 689-693. DOI: 10.1109/ICIG.2007.39

Yang, F., B. Ma, Q.X. Wang and D. Yao and F. Chenyan, 2007. Information fusion of biometrics based-on fingerprint, Hand-geometry and Palmprint. Proceedings of the Workshop on Automatic Identification Advanced Technologies, Jun. 7-8, IEEE Xplore Press, Alghero, pp: 247-252. DOI: 10.1109/AUTOID.2007.380628

Yanxia, W. and R. Qiuqi, 2006. Kernel fisher discriminant analysis for palmprint recognition. Proceedings of the 18th International Conference on Pattern Recognition, IEEE Xplore Press, Hong Kong, pp: 457-460. DOI: 10.1109/ICPR.2006.737

Yazdanpanah, A.P., K. Faez and R. Amirfattahi, 2010. Multimodal biometric system using face, ear and gait biometrics. Proceedings of the Information Sciences Signal Processing and their Applications, May 10-13, IEEE Xplore Press, Kuala Lumpur, pp: 251-254. DOI: 10.1109/ISSPA.2010.5605477

Zhonghua, L. and L. Bibo, 2010. Iris recognition method based on the coefficients of morlet wavelet transform. Proceedings of the International Conference on Intelligent Computation Technology and Automation, May 11-12, IEEE Xplore Press, Changsha, pp: 576-580.

DOI: 10.1109/ICICTA.2010.783 